\title{
Using Microgravity at the Inernational Space Station to Lead to New Therapeutics for Taspase1: A Novel Cancer Target
}

\author{
Jose Martin-Garcia ${ }^{1}$, Nirupa Nagaratnam ${ }^{1}$, Rebecca Jernigan'1, Jay-How Yang1 ${ }^{1}$ Michele Zacks ${ }^{1}$, April \\ Spinale ${ }^{2}$, Marc Giulianotti2 ${ }^{2}$ Barbara Mroczkowski ${ }^{3}$, Andrew Flint ${ }^{3}$, Petra Fromme ${ }^{1}$
}

1. Center for Applied Structural Discovery, Biodesign Institute, Arizona State University, Tempe, AZ

2. The Center for the Advancement of Science in Space (CASIS), Melbourne, FL

3. Frederick National Lab for Cancer Research, National Cancer Institute, National Institutes of Health, Frederick, MD Email Contact: jose.martingarcia@asu.edu

Taspase1 (threonine aspartase 1) is an enzyme that breaks peptide bonds in some amino acids; these enzymes are also known as endopeptidases. Taspase1 is overexpressed in primary human cancers. It has been identified as a potentially potent anticancer drug target because loss of Taspase 1 activity disrupts proliferation of human cancer cells in vitro and in mouse tumor xenograft models of glioblastoma. Based on its promise as a novel anti-cancer therapeutic target, Taspase1 is accepted into the National Cancer Institute (NCI) Experimental Therapeutics (NExT) Chemical Biology Consortium (CBC) drug discovery and development pipeline. A high-resolution structure of the enzymatically active full-length Taspase1, and Taspase1 in complex with substrate and inhibitors greatly enables optimization (through rational design) of pharmacologically potent Taspase 1 inhibitors.

Crystallographic structures of a truncated version of Taspase1 have been determined as apo enzyme and in complex with covalent inhibitors; however, the truncated form of the enzyme is catalytically inactive. The full-length enzyme contains an additional sequence that is anticipated to form a helical structure near the catalytic site. Crystals of the active, full-length Taspase 1 have been grown and its structure determined at synchrotron sources to $3.5 \AA$ A resolution. Despite the low resolution, we could identify the helical fragment near the active site predicted to be involved with substrate binding. However, crystals show an extremely high degree of imperfection, which hamper the ability to obtain a high-resolution structure of Taspase1.

Crystal growth in a microgravity environment has previously been demonstrated to improve the quality of protein crystals enhancing the arrangement of molecules to produce crystals that are more optimal than those grown on the ground. Thus, the investigation held in microgravity at the International Space Station (Investigation Program CASIS PCG 18, https://www.nasa.gov/mission_pages/station/research/experiments/2968.html), will focus on obtaining highquality crystals that allow us to determine the first high-resolution structure of the Taspase 1 as apo enzyme and with inhibitors. These structures greatly benefit the structure-guided medicinal chemistry of Taspase1 inhibitors as anticancer therapeutics.

\section{Acknowledgement:}

This project has been funded with Federal funds from the National Cancer Institute, National Institutes of Health, under Chemical Biology Consortium Contract No. HHSN261200800001E, The Center for the Advancement of Science in Space (CASIS) and The National Aeronautics and Space Administration (NASA). The content of this publication does not necessarily reflect the views or policies of the Department of Health and Human Services, nor does mention of trade names, commercial products, or organizations imply endorsement by the U.S. Government 